\title{
Atrofia muscular espinal y bulbar: enfermedad de Kennedy. Aspectos clínicos y genéticos
}

\author{
Mariana Moraes*, Judith Calvo ${ }^{\dagger}$, Nélida Montano ${ }^{\ddagger}$, \\ Ximena Rodríguez ${ }^{\S}$, Roberto Quadrelli`, Alicia Vaglio**
}

\section{Resumen}

La atrofia muscular espinal y bulbar es una enfermedad neurológica caracterizada por degeneración gradual de la motoneurona inferior, que resulta en debilidad muscular, atrofia y fasciculaciones. Es una entidad de etiología genética con mecanismo de herencia ligado al cromosoma $X$ recesivo, por lo que afecta a varones, en la que se produce una expansión del triplete CAGn en el gen del receptor de andrógenos. Se manifiesta por signos de insensibilidad a los andrógenos (ginecomastia e infertilidad). A partir de los 20-30 años, aproximadamente, comienzan los signos de afectación de la motoneurona inferior a nivel espinal con calambres y temblor de acción y posteriormente debilidad muscular. En la evolución se evidencia compromiso bulbar. Se presenta el caso clínico-genealógico de un varón de 32 años con temblores en quien se confirma molecularmente la enfermedad de Kennedy. Este es el primer caso, hasta nuestro conocimiento, reportado en Uruguay. Se destaca la importancia de plantear dicha afección en un paciente joven con "temblores" cuando aún no es ostensible la debilidad muscular. La historia familiar es de capital importancia. La presencia de fasciculaciones en el estudio eléctrico a nivel perioral es muy sugestiva de esta patología. La confirmación molecular es importante para el asesoramiento genético.

Palabras clave: Atrofia bulboespinal ligada al $\mathrm{X}$

Enfermedad de Kennedy

Informes de casos

Key words: Bulbo-spinal atrophy, X-linked

Kennedy disease

Case reports

\footnotetext{
* Especialista en Pediatría. Especialista en Terapia intensiva pediátrica. Experto universitario en Genética médica: Escuela Europea de Ciencias, centro adscrito a la Universidad Rey Juan Carlos, Valencia, España. Instituto de Genética Médica, Hospital Italiano, Uruguay.

† Especialista en Neurología. Diplomada en Neurofisiología Clínica (electro diagnóstico). Ex Asistente de Neurología (Instituto de Neurología, Hospital de Clínica-UDELAR). Neuróloga en Asociación Española y en HCFFAA.

‡ Especialista en Pediatría. Especialista en Neonatología. Ex Asistente en Neonatología Facultad de Medicina-UDELAR. Experto universitario en Genética médica: Universitat de Valencia. Instituto de Genética Médica, Hospital Italiano. Uruguay.

$\S$ Doctor en Medicina. Residente de Pediatría. Experto universitario en Genética médica: Universitat de Valencia. Instituto de Genética Médica, Hospital Italiano. Uruguay.

I Genetista. Ex Profesor Agregado del Departamento de Genética médica de la Facultad de Medicina-UDELAR. Instituto de Genética Médica, Hospital Italiano. Uruguay. Académico titular, Academia Nacional de Medicina, Montevideo,Uruguay.

** Especialista en Pediatría. Genetista. Director técnico del Instituto de Genética Médica, Hospital Italiano. Uruguay.

Correspondencia: Dra. Mariana Moraes. Ayacucho 3386 bis. Montevideo, Uruguay. Correo electrónico: marianamoraes_16@hotmail.com

Los autores declaran no tener conflicto de intereses.

Recibido: 18/12/18

Aprobado: $20 / 5 / 18$
} 


\section{Introducción}

La atrofia muscular espinal y bulbar (AMEB) es una rara enfermedad neuromuscular lentamente progresiva en la que se produce una degeneración gradual de la motoneurona inferior, que resulta en debilidad muscular, atrofia y fasciculaciones ${ }^{(1,2)}$. Fue descrita por primera vez en 1968 por Kennedy y colaboradores en 11 varones de dos familias relacionadas ${ }^{(3)}$. Se estima una prevalencia de 1 cada 300.000 varones $^{(1)}$, si bien esta varía mucho en función de la región analizada, presentando una de las mayores prevalencias en Finlandia, donde es la enfermedad de motoneurona inferior más prevalente, incluso más frecuente que la esclerosis lateral amiotrófica $(E L A)^{(4)}$. A la vez, su prevalencia podría estar subestimada dado que el diagnóstico muchas veces se confunde con el de otras enfermedades neuromusculares ${ }^{(5)}$.

Es una enfermedad hereditaria ligada al cromosoma $\mathrm{X}$ recesiva, por lo que afecta a varones ${ }^{(1)}$, en la que se produce una expansión del triplete CAGn (poliglutamina) en el exón 1 del gen del receptor de andrógenos (RA) en el cromosoma X (Xq11-q12) ${ }^{(1,2)}$. Esta fue la primera enfermedad en la que se describió el mecanismo molecular por expansión de tripletes ${ }^{(2)}$.

El RA es un receptor intracelular que en ausencia de ligando (testosterona y dihidrotestosterona) se localiza en el citoplasma unido a proteínas de choque térmico. En presencia de ligando, el receptor se disocia del complejo y se dirige al núcleo donde actúa como un factor de transcripción de múltiples genes. La expansión de la poliglutamina causa tanto pérdida como ganancia de la función del RA. La pérdida de función se evidencia en los síntomas de insensibilidad a andrógenos. Sin embargo, el efecto principal es una ganancia tóxica de la función en las neuronas motoras y en el músculo ${ }^{(2)}$.

Clínicamente se caracteriza por su comienzo en la adolescencia con signos de insensibilidad a los andrógenos como ginecomastia y posteriormente infertilidad. A partir de los 20-30 años, aproximadamente, comienzan los signos de afectación de la motoneurona inferior a nivel espinal con debilidad muscular, calambres y temblor de acción. Posteriormente agregan compromiso bulbar con fasciculaciones de lengua, labios o región perioral, disartria y alteraciones en la deglución. Luego de 10 o 20 años del inicio de los síntomas muchos de los afectados agregan dificultad para subir escaleras y con el tiempo amiotrofia proximal y ocasionalmente distal ${ }^{(1)}$. Puede evidenciarse una participación del sistema nervioso sensitivo y endócrino (diabetes mellitus y dislipemias) ${ }^{(6)}$. No se observan signos de compromiso de motoneurona superior como espasticidad e hiperreflexia ${ }^{(1-6)}$.

El diagnóstico se confirma mediante la identificación de la expansión (más de 35 repeticiones) en hetero- cigosis del triplete CAG en el gen AR del cromosoma $X^{(1)}$.

Dado que es una enfermedad ligada al cromosoma $X$ recesiva afecta a varones, y si estos son fértiles, todas sus hijas mujeres serán portadoras de la enfermedad y ninguno de sus hijos varones estará enfermo. Las mujeres portadoras tienen un $50 \%$ de probabilidades de transmitir la expansión del trinucleótido a sus hijos. Los hijos varones que hereden la mutación de sus madres serán enfermos y las hijas serán portadoras no afectadas ${ }^{(1)}$.

Se presenta el caso clínico de un varón de 32 años que consulta por temblores en quien se confirma molecularmente la enfermedad de Kennedy. Este es el primer caso, hasta nuestro conocimiento, reportado en Uruguay.

\section{Caso clínico}

$\mathrm{BC}$, sexo masculino, 32 años, escribano, con antecedentes personales de ginecomastia diagnosticada a los 12 años. Historia de fatiga, artralgias y mialgias posejercicio. Del análisis genealógico surge que el paciente es producto de cuarto embarazo de una pareja no consanguínea. Madre de 62 años portadora de debilidad, calambres y parestesias en manos interpretados como síndrome de túnel carpiano bilateral. Hermano de 38 años presenta temblores de cara y miembros; tiene dos hijos (un varón y una niña) productos de fertilización in vitro (FIV) debido a infertilidad en la pareja. Otro hermano de 35 años también presenta temblores a predominio de hemicara inferior. El paciente tiene un hijo de 4 años sano.

Enfermedad actual: a partir de los 20 años refiere temblor de inicio en manos, luego en cara, sobre todo a nivel perioral, que se fue acentuando. En la evolución agrega calambres y desde los 30 años, aproximadamente, tropiezos frecuentes y fasciculaciones a predominio de cuádriceps, gemelos y músculos de antebrazos. Niega dificultad para subir escaleras, disartria o alteraciones en la deglución. Algunos meses previos a la consulta, instala hipoestesia en calcetín y parestesias en manos. Dicha sintomatología motiva el abandono de la actividad física y le genera gran dificultad en el ejercicio de su actividad profesional.

Al examen se destaca: paciente con temblor de miembros, fino, rápido, de acción y postural, temblor fino mandibular. Motilidad ocular extrínseca normal. Durante examen, calambre de músculos maseteros. Temblor de lengua y úvula. En el sector espinal no se evidencia amiotrofia. Fuerzas conservadas. Reflejos osteotendinosos disminuidos a predominio de miembros inferiores. Hipoestesia en calcetín. No ataxia. No dismorfias.

En noviembre de 2017, luego de consulta con neuróloga, se plantea enfermedad de Kennedy e inicia tratamiento con propranolol. 
De los estudios realizados se destaca: resonancia magnética de cráneo y columna cervical normal. Estudio eléctrico evidencia neuroconducción motora y sensitiva normal. Signos neurogénicos crónicos en el electromiograma (EMG) con una distribución distal y proximal en los miembros, con fasciculaciones universales que en el sector craneal se registran en borla del mentón.

Es referido al Instituto de Genética para confirmación molecular del diagnóstico. Se realiza el análisis de la expansión del triplete CAGn en el gen AR, confirmándose el diagnóstico de AMEB. Se realiza asesoramiento genético y se sugiere estudio molecular en ambos hermanos. Su hermano de 35 años se realiza estudio que también confirma la enfermedad.

\section{Discusión}

El paciente presenta un cuadro clínico característico. La ginecomastia es un signo frecuente aunque no constante $^{(7)}$ que se presenta en aproximadamente $73 \%$ de los pacientes ${ }^{(8)}$.

Tal como lo describió Kennedy en 1968 y otros autores posteriormente, los calambres y el temblor, sobre todo en manos, preceden a la debilidad muscular. En el paciente la debilidad muscular no es aún ostensible, ya que se manifiesta entre la tercera a quinta década de la vida. El temblor, que es de características similares al temblor esencial, está presente en el $80 \%$ de los pacientes y su causa es desconocida ${ }^{(3,8,9)}$. Tal como se ha reportado en este caso, un signo característico en esta patología son las fasciculaciones en la región perioral ${ }^{(1-9)}$. Las fasciculaciones y amiotrofia de lengua predicen la aparición de los síntomas bulbares (disartria y disfagia) ${ }^{(9)}$.

El compromiso sensitivo ha sido descrito por otros autores ${ }^{(9,10)}$ y sugiere que no se produce solamente una degeneración primaria de las neuronas del asta anterior, sino también un compromiso del nervio debido a la degeneración neuronal $^{(10)}$.

En general, la afectación sensitiva es un hallazgo del estudio eléctrico sin una expresión clínica mayor. Sin embargo, el paciente presenta en el momento del diagnóstico un síndrome sensitivo deficitario distal, bilateral y simétrico en miembros inferiores, pese a que las amplitudes de sus potenciales de acción sensitivos en el estudio eléctrico estuvieron dentro de límites normales. Esto se explica por una gran variabilidad interindividual en los valores considerados normales de amplitud de los potenciales sensitivos y nos permite concluir que los valores hallados, si bien dentro de rangos normales, están descendidos para el paciente.

Otra alteración endocrinológica asociada a la insensibilidad a andrógenos, como la infertilidad, no se evidencia en el paciente ${ }^{(8)}$.
Si bien en el caso reportado el diagnóstico fue realizado precozmente con signos menos específicos como la ginecomastia y el temblor de acción, en ausencia de debilidad ostensible, realizar un diagnóstico precoz puede ser difícil ${ }^{(5)}$. En presencia de debilidad muscular y un síndrome de motoneurona inferior el diagnóstico diferencial debe realizarse con otras enfermedades motoneuronales. Entre estas la asociación o no de signos de motoneurona superior y el curso evolutivo son los elementos clínicos de mayor utilidad en el razonamiento diagnóstico.

La ELA es una enfermedad motoneuronal de progresión rápida en la que hay compromiso de ambas motoneuronas y no asocia manifestaciones endocrinológicas vinculadas a insensibilidad a andrógenos ${ }^{(5)}$. La atrofia muscular primaria es una variante en donde no hay compromiso de la motoneurona superior y su diferenciación de la enfermedad de Kennedy puede ser más difícil al momento de la presentación. De la misma manera, las atrofias musculares espinales de inicio en el adulto forman parte del diagnóstico diferencial de la entidad que nos ocupa. En estas también puede haber un temblor de acción, no hay compromiso de la motoneurona superior ni ginecomastia. Los signos de motoneurona inferior en estos casos no involucran el sector bulbar ni fascial y ese es otro elemento que las diferencia de la enfermedad de Kennedy ${ }^{(11)}$.

El diagnóstico se confirma mediante el estudio molecular de la expansión del triplete CAGn (mayor a 35 repeticiones) en el gen AR. La expresión fenotípica es variable incluso dentro de una misma familia e independiente del tamaño de la expansión ${ }^{(5-12)}$. Otros autores postulan una relación inversamente proporcional entre el tamaño de la expansión y la edad de inicio de los sínto$\operatorname{mas}^{(5-13)}$. No se ha descrito fenómeno de anticipación genética $^{(12)}$.

Cabe destacar que si bien es una patología que afecta clínicamente a varones, pueden observarse síntomas más sutiles en mujeres portadoras ${ }^{(1)}$.

Actualmente no existe un tratamiento específico para la AMEB. Este es sintomático y consiste en fisioterapia y rehabilitación ${ }^{(5)}$. El temblor puede beneficiarse del tratamiento con propranolol ${ }^{(9)}$. En casos de ginecomastia es posible realizar cirugía de reducción mamaria. Se deben prevenir las complicaciones secundarias, entre ellas la neumonía aspirativa debido a debilidad bul$\operatorname{bar}^{(1-14)}$.

Teniendo en cuenta que el AR es ligando dependiente y que ejerce su función en presencia de andrógenos ${ }^{(2)}$, se postuló una estrategia terapéutica basada en la reducción de andrógenos. En este sentido, Banno y colaboradores realizaron un ensayo clínico aleatorizado multicéntrico controlado con placebo en el que la leuproreli- 
na, que suprime la secreción de testosterona, no mostró un efecto definitivo sobre las funciones motoras, aunque se evidenció una mejoría de la función deglutoria en un subgrupo de pacientes cuya enfermedad fue menor a diez años ${ }^{(15)}$. Por todo lo expuesto, no existe hasta el momento un consenso en cuanto a la indicación de antiandrógenos para el tratamiento de las manifestaciones neurológicas.

$\mathrm{Al}$ igual que en otras enfermedades neurodegenerativas poliglutamina (causadas por la expansión del triplete CAGn) como la corea de Huntington y la ataxia espinocerebelosa, la desregulación transcripcional y las alteraciones postraduccionales se han convertido en el blanco de la investigación para nuevos tratamien$\operatorname{tos}^{(16)}$. Al respecto, Minamiyama y colaboradores demostraron, en modelo animal de AMEB, que un inhibidor de la histona deacetilasa (HDAC) mejora el fenotipo neurológico ${ }^{(17)}$. Montie $\mathrm{H}$ y colaboradores demostraron en modelos de cultivo celular que el Sirt1, una clase de HDAC, es neuroprotectora al desacetilar el AR en lisinas 630/632/633 $3^{(18)}$.

Otra línea de investigación basada en los hallazgos de que los pacientes con esta enfermedad tienen bajas concentraciones de factor de crecimiento insulino símil (IGF-1) se centra en la sobreexpresión y administración de IGF-1. Al respecto, Grunseich y colaboradores evaluaron la seguridad, la tolerabilidad y la eficacia de BVS857, un mimético de IGF-1, en pacientes con atrofia muscular espinal y bulbar con concentraciones bajas de IGF-1. En este ensayo aleatorizado, doble ciego, controlado con placebo, se demostró que este fármaco es seguro, sin efectos adversos graves. Si bien no hubo diferencias en las medidas de fuerza muscular y función entre los grupos, los participantes que recibieron BVS857 no perdieron volumen muscular del muslo respecto al grupo placebo. Por todo lo antedicho, podrían ser necesarios estudios adicionales para evaluar la eficacia de la activación de la vía de IGF-1 en esta enfermedad ${ }^{(19)}$.

La expectativa de vida es normal o ligeramente reducida $^{(1-5)}$, siendo la neumonía aspirativa y el fallo respiratorio debido al compromiso bulbar el principal problema al que se ven enfrentados estos pacientes ${ }^{(1-13)}$, pero estas complicaciones se ven en una minoría de los pacientes y en etapas muy evolucionadas de la enfermedad.

Sin embargo, como lo demostró Guber R y colaboradores en un estudio que evaluó los síntomas de la enfermedad desde la perspectiva de los pacientes y su impacto en la calidad de vida, se demostró que los pacientes alteran su estilo de vida para adaptarse a la progresión de la enfermedad. La debilidad muscular y las afecciones de la esfera psicológica fueron de los más mencionados por los pacientes. Deberíamos evaluar estos síntomas "no físicos" en la consulta clínica, ya que podrían requerir una intervención ${ }^{(20)}$.

Se sugiere, además, realizar control de niveles de glicemia y perfil lipídico, ya que presentan un aumento en la incidencia de diabetes mellitus y dislipemias ${ }^{(8-10)}$. También se recomienda evaluación cardiológica con electrocardiograma, pues se ha reportado síndrome de Brugada en pacientes con esta patología. Se cree que podría deberse a la acumulación de la proteína del RA mutada en el miocardio ${ }^{(21)}$. Además, se sugiere evaluación de la función pulmonar anual en casos avanzados ${ }^{(1)}$.

Se destaca la importancia de la intervención del equipo multidisciplinario con la participación de neurólogo, médico internista, cardiólogo, psicólogo, neumólogo y genetista. En relación con el abordaje genético se destaca la confirmación diagnóstica y el asesoramiento genético personal y familiar que permite identificar otros afectados y ofrecerles eventual diagnóstico genético preimplantacional.

\section{Conclusiones}

La AMEB es una patología poco frecuente, no obstante es importante considerarla ante un paciente joven de sexo masculino que presenta "temblor" aun en ausencia de debilidad muscular. La presencia clínica o en el estudio eléctrico de fasciculaciones a nivel perioral es muy característica. El análisis genealógico reviste capital importancia en la identificación de madres portadoras de la patología con síntomas leves, así como otros varones afectados emparentados por vía materna. La confirmación molecular es importante para el asesoramiento genético y eventual diagnóstico prenatal.

\section{Abstract}

Spinal and bulbar muscular atrophy (SBMA) is a neurological disease characterized by the progressive degeneration of the inferior motor neurones, what results in muscle weakness, atrophy and fasciculations. It possesses a genetic etiology with X-linked recessive inheritance mode, and thus affects men. There is an an abnormal expansion of the CAG polyglutamine encoding repeat within the androgen receptor gene. It is noticed by signs of androgen insentistivity (gynecomastia and infertility). At 20-30 years old approximately signs of compromise of the lower motor neurones in the spine are seen in cramps and action tremor followed by muscle weakness, evidencing bulbar involvement in the evolution. The study presents the ciínical-genealogical case of a 32 year-old male with tremor, whose Kennedy disease was confirmed with molecules. This is the first case reported in Uruguay as far as we know.

The importance of considering this condition is pointed out in a young patient with "tremor" when mus- 
cle weakness is not evident yet. Family history is key. The presence of fasciculation in the electrical study strongly suggests this condition. Molecular confirmation is important for genetic advice purposes.

\section{Resumo}

A atrofia muscular bulbo-espinal (BSMA) é uma doença neurológica caracterizada pela degeneração gradual do neurônio motor inferior causando fraqueza muscular, atrofia e fasciculações. É uma entidade de etiologia genética com mecanismo de herança ligada ao cromossoma $\mathrm{X}$ recessivo, afetando por isso a indivíduos do sexo masculino, nos quais se observa a expansão do triplete CAGn no gene do Receptor de Andrógenos (RA). Manifesta-se pela ausência de sensibilidade aos andrógenos (ginecomastia e infertilidade); a partir dos 20-30 anos aproximadamente começam a manifestar-se os sinais de afetação do neurônio motor inferior na região espinal com câimbras e tremor de ação e posteriormente debilidade muscular. Em sua evolução observa-se compromisso bulbar. Apresenta-se o caso clínico - genealógico de um indivíduo de sexo masculino de 32 anos com tremores, no qual foi realizado diagnóstico molecular de doença de Kennedy. Este é o primeiro caso informado no Uruguai, que seja de nosso conhecimento. Destaca-se a importância da suspeita desta afecção em um paciente jovem com "tremores" mesmo quando a debilidade muscular ainda não é ostensível. A história familiar é fundamental. A presença de fasciculações no estudo elétrico na região perioral é muito sugestiva desta patologia. A confirmação molecular é importante para o assessoramento genético.

\section{Bibliografía}

1. La Spada A. Spinal and bulbar muscular atrophy. En: Adam MP, Ardinger HH, Pagon RA, Wallace SE, Bean LJH, Stephens K, et al., eds. GeneReviews. Seattle (WA): University of Washington, Seattle, 1993-2018. Disponible en: https://www.ncbi.nlm.nih.gov/books/NBK1333/?report=reader [Consulta: 12 mar. 2018].

2. Fischbeck KH. Spinal and bulbar muscular atrophy overview. J Mol Neurosci 2016; 58(3):317-20.

3. Kennedy WR, Alter M, Sung JH. Progressive proximal spinal and bulbar muscular atrophy of late onset. A sex-linked recessive trait. Neurology 1968; 18(7):671-80.

4. Udd B, Juvonen V, Hakamies L, Nieminen A, Wallgren-Pettersson C, Cederquist K, et al. High prevalence of Kennedy's disease in Western Finland-is the syndrome underdiagnosed? Acta Neurol Scand 1998; 98(2):128-33.

5. Fratta $\mathbf{P}$, Nirmalananthan $\mathbf{N}$, Masset L, Skorupinska I, Collins T, Cortese A, et al. Correlation of clinical and molecular features in spinal bulbar muscular atrophy. Neurology 2014; 82(23):2077-84.
6. Chen Y, Luo P, Li Z, Hu H, Wu D, Xu T, et al. Kennedy disease with difficulty in differential diagnosis: a case report. Medicine (Baltimore) 2017; 96(19):e6792.

7. Stefanis C, Papapetropoulos T, Scarpalezos S, Lygidakis G, Panayiotopoulos CP. X-linked spinal and bulbar muscular atrophy of late onset. A separate type of motor neuron disease? J Neurol Sci 1975; 24(4):493-503.

8. Dejager S, Bry-Gauillard H, Bruckert E, Eymard B, Salachas F, LeGuern E, et al. A comprehensive endocrine description of Kennedy's disease revealing androgen insensitivity linked to CAG repeat length. J Clin Endocrinol Metab 2002; 87(8):3893-901.

9. Harding AE, Thomas PK, Baraitser M, Bradbury PG, Morgan-Hughes JA, Ponsford JR. X-linked recessive bulbospinal neuronopathy: a report of ten cases. J Neurol Neurosurg Psychiat 1982; 45(11):1012-9.

10. Wilde J, Moss T, Thrush D. X-linked bulbo-spinal neuronopathy: a family study of three patients. J Neurol Neurosurg Psychiat 1987; 50(3):279-84.

11. Fernandes Filho JA, Ubogu EE. Atypical motor neuron disorders. En: Katirji B, Kaminski HJ, Ruff RL, eds. Neuromuscular disorders in clinical practice. 2nd ed. New York: Springer, 2014:441-66.

12. Amato AA, Prior TW, Barohn RJ, Snyder P, Papp A, Mendell JR. Kennedy's disease: a clinicopathologic correlation with mutations in the androgen receptor gene. Neurology 1993; 43(4):791-4.

13. Atsuta N, Watanabe H, Ito M, Banno H, Suzuki K, Katsuno M, et al. Natural history of spinal and bulbar muscular atrophy (SBMA): a study of 223 japanese patients. Brain 2006; 129:1446-55.

14. Alves CN, Braga TKK, Somensi DN, Nascimento BSVD, Lima JAS, Fujihara S. X-linked spinal and bulbar muscular atrophy (Kennedy's disease): the first case described in the Brazilian Amazon. Einstein (Sao Paulo) 2018; 16(2):eRC4011.

15. Katsuno M, Banno H, Suzuki K, Hashizume A, Adachi H, Tanaka F, et al. Anti-androgen therapy for spinal and bulbar muscular atrophy (SBMA). Rinsho Shinkeigaku 2012; 52(11):1207-9.

16. Xiang C, Zhang S, Dong X, Ma S, Cong S. Transcriptional dysregulation and post-translational modifications in polyglutamine diseases: from pathogenesis to potential therapeutic strategies. Front Mol Neurosci 2018; 11:153.

17. Minamiyama M, Katsuno M, Adachi H, Waza M, Sang Ch, Kobayashi Y, et al. Sodium butyrate ameliorates phenotypic expression in a transgenic mouse model of spinal and bulbar muscular atrophy. Hum Mol Genet 2004; 13:1183-92.

18. Montie H, Pestell R, Merry D. SIRT1 modulates aggregation and toxicity through deacetylation of the androgen receptor in cell models of SBMA. J Neurosci 2011; 31:17425-36.

19. Grunseich Ch, Miller R, Swan T, Glass DJ, Mouelhi ME, Fornaro M, et al. Safety, tolerability, and preliminary efficacy of an IGF-1 mimetic in patients with spinal and bulbar muscular atrophy: a randomised, placebo-controlled trial. 
Lancet Neurol 2018; 17(12):1043-52. doi: 10.1016/ S1474-4422(18)30320-X.

20. Guber RD, Kokkinis AD, Schindler AB, Bendixen RM, Heatwole CR, Fischbeck KH, et al. Patient-identified im- pact of symptoms in spinal and bulbar muscular atrophy. Muscle Nerve 2018; 57(1):40-4.

21. Araki A, Katsuno M, Suzuki K, Banno H, Suga N, Hashizume A, et al. Brugada syndrome in spinal and bulbar muscular atrophy. Neurology 2014; 82(20):1813-21.

\section{Contribución de autores}

Mariana Moraes, https://orcid.org/0000- 0001-7555-0857. Revisión de la literatura y redacción. Judith Calvo, https://orcid.org/0000-0001-5214-5405. Revisión crítica.

Nélida Montano, https://orcid.org/0000-0002-8780-1745. Revisión crítica.

Ximena Rodriguez, https://orcid.org/0000-0003-2502-4749. Revisión crítica.

Roberto Quadrelli, https://orcid.org/0000-0003-2144-8296. Revisión crítica.

Alicia Vaglio, https://orcid.org/0000-0002-0610-0606. Revisión crítica. 